\title{
Quantitative proteomic analysis reveals concurrent RNA-protein interactions and identifies new RNA-binding proteins in Saccharomyces cerevisiae
}

\author{
Daniel M. Klass, ${ }^{1,2}$ Marion Scheibe, ${ }^{3}$ Falk Butter, $^{3}$ Gregory J. Hogan, ${ }^{1,2}$ \\ Matthias Mann, 3,4 and Patrick O. Brown ${ }^{1,2,4}$ \\ ${ }^{7}$ Department of Biochemistry, Stanford University School of Medicine, Stanford, California 94305, USA; ${ }^{2}$ Howard Hughes Medical \\ Institute, Stanford University School of Medicine, Stanford, California 94305, USA; ${ }^{3}$ Department of Proteomics and Signal \\ Transduction, Max Planck Institute for Biochemistry, 82152 Martinsried, Germany
}

\begin{abstract}
A growing body of evidence supports the existence of an extensive network of RNA-binding proteins (RBPs) whose combinatorial binding affects the post-transcriptional fate of every mRNA in the cell-yet we still do not have a complete understanding of which proteins bind to mRNA, which of these bind concurrently, and when and where in the cell they bind. We describe here a method to identify the proteins that bind to RNA concurrently with an RBP of interest, using quantitative mass spectrometry combined with RNase treatment of affinity-purified RNA-protein complexes. We applied this method to the known RBPs Pabl, Nab2, and Puf 3. Our method significantly enriched for known RBPs and is a clear improvement upon previous approaches in yeast. Our data reveal that some reported protein-protein interactions may instead reflect simultaneous binding to shared RNA targets. We also discovered more than 100 candidate RBPs, and we independently confirmed that $77 \%$ (23/30) bind directly to RNA. The previously recognized functions of the confirmed novel RBPs were remarkably diverse, and we mapped the RNA-binding region of one of these proteins, the transcriptional coactivator Mbfl, to a region distinct from its DNA-binding domain. Our results also provided new insights into the roles of $\mathrm{Nab} 2$ and Puf 3 in post-transcriptional regulation by identifying other RBPs that bind simultaneously to the same mRNAs. While existing methods can identify sets of RBPs that interact with common RNA targets, our approach can determine which of those interactions are concurrent-a crucial distinction for understanding post-transcriptional regulation.
\end{abstract}

[Supplemental material is available for this article.]

Life depends on the coordinated temporal, spatial, and stoichiometric regulation of gene expression. Combinatorial binding by specific transcription factors allows for the concerted temporal regulation of large sets of genes in physiological and developmental programs at a transcriptional level. The resulting RNA transcripts are also subject to further regulation at the levels of RNA processing, transport, localization, translation, and degradation. The added dimensions of regulation provided by RNAbinding proteins (RBPs) enable more precise temporal, spatial, and stoichiometric control of protein production (Wang et al. 2002; Paquin et al. 2007; Jansen et al. 2009; Kurischko et al. 2011). Specific RBPs bind to distinct sets of mRNAs, typically encoding proteins destined for similar subcellular localizations or with related biological functions, suggesting a model in which concerted, combinatorial binding of specific mRNAs by specific sets of RBPs can affect the post-transcriptional fate of potentially every mRNA in the cell (Hieronymus and Silver 2003; Gerber et al. 2004; Ong et al. 2004; Keene 2007a,b; Hogan et al. 2008). Despite the many lines of evidence pointing to pervasive posttranscriptional regulation of gene expression mediated by RBPs, we still do not have a complete understanding of which proteins

\footnotetext{
${ }^{4}$ Corresponding authors

E-mail pbrown@stanford.edu

E-mail mmann@biochem.mpg.de

Article published online before print. Article, supplemental material, and publication date are at http://www.genome.org/cgi/doi/10.1101/gr.153031.112. Freely available online through the Genome Research Open Access option.
}

bind to mRNA, which of these bind concurrently, and when and where in the cell they bind.

Previous global approaches to identify proteins that interact with mRNAs in yeast have been mostly focused on in vitro binding, mass spectrometry, or computational predictions. Although powerful, these techniques may miss complex RNA-protein interactions assembled in vivo, less abundant RBPs, and RBPs that lack domains known to bind RNA (Butter et al. 2009; Scherrer et al. 2010; Tsvetanova et al. 2010). In fact, >75\% (503 out of 647) of the proteins annotated as RBPs lack domains known to bind RNA (Tsvetanova et al. 2010). Conversely, despite the fact that $\sim 10 \%$ of the yeast proteome is annotated as "known" RBPs (annotated in the yeast genome database, experimentally validated, or with homology with known RNA-binding domains), some proteins not annotated as RBPs nonetheless reproducibly copurify with distinct sets of RNAs in vivo (Hogan et al. 2008). The known functions of some RBPs would not suggest their involvement in the post-transcriptional regulation of RNA. For example, the metabolic enzyme aconitase, which catalyzes the isomerization of citrate to isocitrate, also functions as an RNA-binding protein, binding to iron regulatory elements in target mRNAs to regulate their translation or stability in response to iron availability (Hentze et al. 1987a,b; Casey et al. 1988; Leibold and Munro 1988; Rouault et al. 1989; Bertrand et al. 1993). Previous work using protein microarrays to search for new RNA-binding proteins in yeast identified additional unexpected RBPs, including several enzymes (Scherrer et al. 2010; Tsvetanova et al. 2010). Recently, two papers used mass spectrometry to identify hundreds of novel 
RBPs in human cells (Baltz et al. 2012; Castello et al. 2012). These and other examples suggesting regulatory RNA-binding activity in unexpected proteins highlight the need for additional experimental methods to enable the quantitative, unbiased, and accurate discovery of novel RNA-protein interactions from complexes assembled in vivo.

The post-transcriptional operon model hypothesizes that the fate of a given mRNA molecule is influenced by the concerted, combinatorial binding of specific RBPs (Keene 2007a,b) yet we know surprisingly little about which RBPs bind to mRNAs concurrently. It is thought that the specific complement of RBPs bound to a given mRNA specifies its post-transcriptional fate, but nearly all existing data are limited to defining pairwise interactions between a single RBP and a single mRNA species. Previous work to identify the mRNA targets bound by individual RBPs has mostly relied on purification of the RBP from a whole-cell lysate followed by analysis of the copurifying mRNAs (Gerber et al. 2004; Ule et al. 2005; Keene 2007a,b; Hogan et al. 2008; Bohnsack et al. 2009; Granneman et al. 2009, 2010; Wolf et al. 2010; Scherrer et al. 2011; Schenk et al. 2012). These approaches do not differentiate between two RBPs that bind simultaneously to their common mRNA targets and two RBPs that bind to a common set of mRNA targets but at different times or in different cellular locations. This limits our understanding of post-transcriptional regulation, because from birth to death the average mRNA molecule is estimated to be bound by at least 10 different known RBPs during the entirety of its processing, export, transport, localization, translation, and degradation (Hogan et al. 2008). The posttranscriptional regulatory network is determined not only by which RBPs bind to a given mRNA, but in what temporal programs and in what combinations with other RBPs. Identifying well-characterized RBPs that bind mRNAs simultaneously with an RBP of unknown role would provide immediate clues to its functions. For example, if an uncharacterized RBP binds concurrently with RBPs known to be involved in splicing, the uncharacterized RBP can be inferred to bind in the nucleus during splicing and possibly play a role in splicing.

Mass spectrometry (MS)-based proteomics is a powerful tool for studying cellular interactions, especially if used in a quantitative format. Stable isotope labeling of amino acids in cell culture, SILAC (Mann 2006), is one such quantitative proteomics technology, and it can be used to detect selective enrichment. This technique has been applied to GFP-tagged proteins (TrinkleMulcahy et al. 2008; Hubner et al. 2010), modified peptides (Schulze and Mann 2004), DNA (Mittler et al. 2009), and RNA (Butter et al. 2009; Baltz et al. 2012; Castello et al. 2012; Scheibe et al. 2012) to identify previously unknown binders. Here we used quantitative mass spectrometry combined with RNase treatment of affinity-purified RNA-protein complexes assembled in vivo to identify the proteins that bind to RNA concurrently with the known RBPs Pab1, Nab2, and Puf3.

\section{Results}

A quantitative proteomic method for identifying RNA-dependent protein interactions

We used quantitative mass spectrometry to identify the proteins that copurify with a protein of interest in an RNA-dependent manner (Fig. 1). We first purified a TAP-tagged protein by IgGprotein-A affinity purification from a "light" (unlabeled) cell lysate and from a "heavy" lysate labeled by incorporation of ${ }^{13} \mathrm{C}$ and

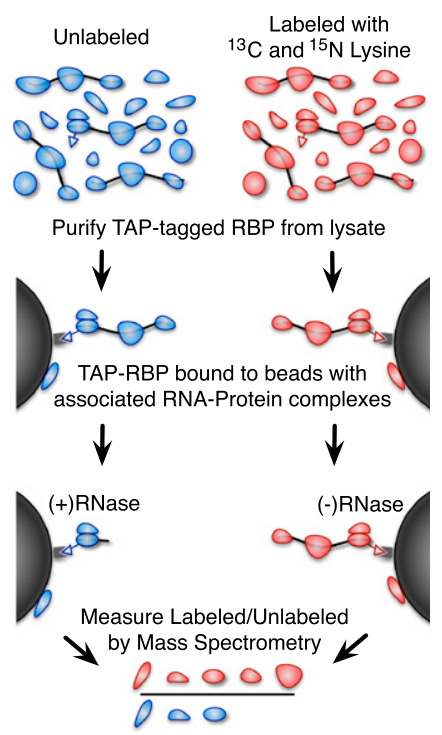

Figure 1. A method for identifying RNA-dependent protein interactions. An overview of our proteomic method for identifying RNAdependent protein interactions. A yeast strain with a TAP-tagged protein of interest is grown in media labeled with heavy $\left({ }^{13} \mathrm{C}\right.$ and ${ }^{15} \mathrm{~N}$ isotope enriched) lysine or unlabeled media. The cells are lysed and the protein of interest is purified using the TAP tag. The unlabeled sample is treated with RNase, and the heavy labeled sample is not. The beads are then boiled in SDS-PAGE buffer to release any bound proteins and combined as heavy labeled RNase untreated and unlabeled treated with RNase (we also performed the inverse as a replicate and to control for labeling-related artifacts). The heavy-to-light ratio measured by mass spectrometry indicates the fraction of the bound protein that was liberated by RNase treatment.

${ }^{15} \mathrm{~N}$ isotope-enriched lysine. We then divided the IgG beads with the associated TAP-tagged protein into two equal parts and digested one of them with RNase. Finally, we combined heavylabeled lysate not treated with RNase with light RNase-treated lysate and quantified the heavy-to-light SILAC ratio by mass spectrometry. By design, this assay specifically measures enrichment due to RNAdependent association with the TAP-tagged protein. The reverse or 'label-swapped' experiment, where instead a heavy-labeled RNasetreated lysate was combined with light (unlabeled) lysate without RNase treatment, served as a replicate and a control for contaminant proteins that are unlabeled in both experiments.

The resulting heavy-to-light SILAC ratios are a measure of the RNA-dependent copurification of a given protein with the TAP-tagged protein of interest. When the heavy labeled sample is not treated with RNase and the light sample is treated with RNase, proteins that are lost from the beads in response to RNase treatment will be present more in the heavy labeled sample than the light sample. Consequently, proteins will tend to have heavyto-light ratios greater than one if they copurify with the TAP-tagged protein of interest in an RNA-dependent manner. For the reverse experiment, in which the heavy labeled sample is treated with RNase and the light sample is not, proteins will have heavy-to-light ratios less than one if they copurify with the TAP-tagged protein of interest in an RNA-dependent manner. To make the results of these replicates directly comparable, we invert the heavy-to-light ratios in the reversed experiment. For simplicity, we represented RNA dependence as the ratio of (-) RNase to (+) RNase, so that RNA-dependent binders would always be expected to have $(-/+)$ RNase ratios greater than one if they copurify with the TAP-tagged 
protein of interest in an RNA-dependent manner, regardless of the labeling scheme.

We used this method to identify proteins that interact in an RNA-dependent manner with the RBPs Pab1, Nab2, or Puf3, respectively. Pab1 and Nab2 have each been shown to bind to more than a thousand different mRNAs, while Puf3 binds to a smaller, highly specific set of mRNAs (Gebauer and Hentze 2004; Gerber et al. 2004; Hogan et al. 2008). To assess the scale and reproducibility of the data, we plotted the RNA dependence of each protein as the $\left(\log _{2}\right)(-/+)$ RNase ratios from the two replicate experiments for Pab1, Nab2, and Puf3 (Fig. 2). In these plots, the reproducible RNA-dependent binders (RDBs) form a tail along the diagonal, while proteins that interact directly with the tagged protein, independent of RNA, are clustered around the origin. As a standard measure of RNA-dependent association with the TAPtagged protein, we first normalized the $(-/+)$ RNase ratios to set the ratio for the TAP-tagged protein itself to one, based on the premise that enrichment of the TAP-tagged protein itself should not be RNA dependent. We then averaged the $(-/+)$ RNase ratios in both replicate experiments and used the base 2 logarithm of this value as our standard measure of RNA-dependent association with the TAP-tagged protein (referred to as RNA-dependence values).

To initially evaluate the performance of this assay, we compared the distribution of RNA-dependence values for proteins annotated as RBPs and proteins without such an annotation (Supplemental Fig. S1). The RNA-dependence values for annotated
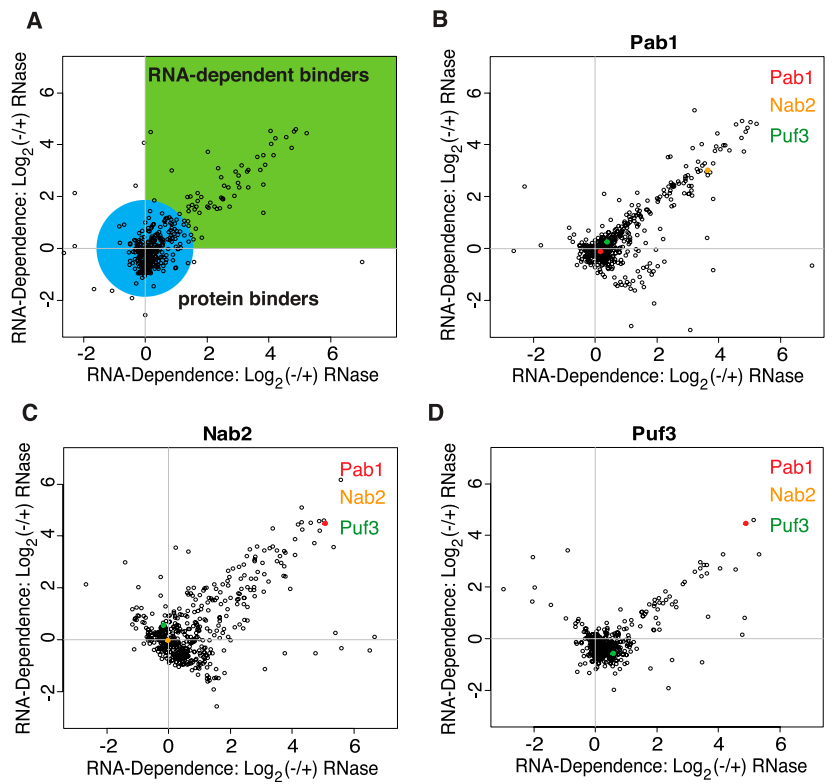

Figure 2. Overview of RNA-dependent interaction data. A scatterplot of the RNA-dependent enrichment values as the log base $2(-/+)$ RNase ratios for two replicates (with inverted labeling schemes) of each protein that we purified. The reproducible RNA-dependent binders form a tail in quadrant 1. $(A)$ A plot of example data with a green box indicating the quadrant where RNA-dependent binders are expected to be found and a blue circle indicating where RNA-independent binders are expected to be found. These colored regions are broad generalizations only and were not used for actual data analysis. (B) A scatterplot of the log base $2(-/+)$ RNase ratios for the replicate experiments with Pab1. The points representing the proteins Pab1, Nab2, and Puf3 are highlighted in red, yellow, and green, respectively. (C) The same scatterplot for experiments with the protein Nab2. (D) The same scatterplot for experiments with the protein Puf3.
RBPs were significantly shifted toward higher values in the Pab1, Nab2, and Puf3 purifications ( $P$-values $4 \times 10^{-8}, 2 \times 10^{-5}$, and $3 \times 10^{-7}$, respectively), showing that the method enables RNAdependent binders (RDBs) to be identified and that proteins with larger RNA-dependence values are more likely to be annotated as RBPs. Although results from traditional mass spectrometry have frequently been biased by protein abundance, we found no correlation between protein abundance and the RNA-dependence values (Supplemental Fig. S2). This demonstrates that our classification of the proteins we detected as RDBs was not affected by their abundance.

To establish a conservative cutoff for the classification of proteins into RNA-dependent and RNA-independent binders, we created a null distribution by modeling RNA-dependence values for proteins with RNA-independent interactions with Pab1, Nab2, and Puf3. To do this, we made two assumptions: first, that after normalization any RNA-dependence values less than zero have a true value of zero and the observed variation from zero is due to noise; and second, that this noise is symmetric about zero (see Methods). We used the null distribution as the basis for estimating an empirical false discovery rate (FDR) for classification of proteins as RDBs, with an FDR threshold of 10\% (Supplemental Fig. S3).

At least half of the proteins classified as RDBs based on our 10\% empirical FDR threshold were proteins known to bind RNA (Fig. 3A). In the combined data set, there were $220 \mathrm{RDBs}, 48 \%$ of which were known RNA-binding proteins. This represents a significant enrichment of known RBPs relative to the set of all proteins that can be detected by mass spectrometry from a yeast whole-cell lysate $\left(\sim 15 \%\right.$, hypergeometric $P$-value $\left.2 \times 10^{-35}\right)$ (Supplemental Table S7; de Godoy et al. 2008). We also examined a published data set of "high-confidence" protein-protein interactions based on large-scale affinity mass spectrometry studies (Gavin et al. 2006; Krogan et al. 2006; Collins et al. 2007), and we discovered that the majority of the previously published physically interacting proteins with Pab1 and Nab2 that we detected in our purifications were actually RNA dependent, suggesting that protein interactions involving RNA-binding proteins (especially those that bind to thousands of different RNAs) may often be indirect and mediated by concurrent binding to RNA molecules (see the Supplemental Material for further information).

The experiments described used a buffer containing EDTA, and we next performed the Pab1 IP experiment in a buffer containing magnesium. This led to a highly significant enrichment of known RNA-binding proteins composed almost entirely of ribosomal proteins and proteins involved in the initiation, elongation, and termination of translation (Supplemental Table S6). The majority of these proteins were not observed as RNA-dependent binders in experiments done in the presence of EDTA, in which ribosomes are no longer assembled on mRNA. These data provide a unique perspective into Pab1-containing RNA-protein complexes involved in translation.

The high frequency of known RBPs among the 220 RDBs identified in this study contrasts with a frequency of $\sim 20 \%$ known RBPs among the 220 highest-ranking hits identified in two previous studies using protein microarrays (including one method developed by members of our group) (Scherrer et al. 2010; Tsvetanova et al. 2010). Despite this difference, our 220 RDBs are significantly enriched in the protein microarray data from Tsvetanova et al. (2010) and also from Scherrer et al. (2010) (Supplemental Fig. S5; Wilcoxon $P$-values 0.005 and 0.009, respectively). However, there was no Spearman rank correlation between 
A

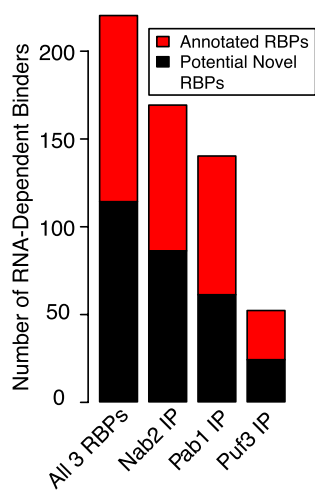

B

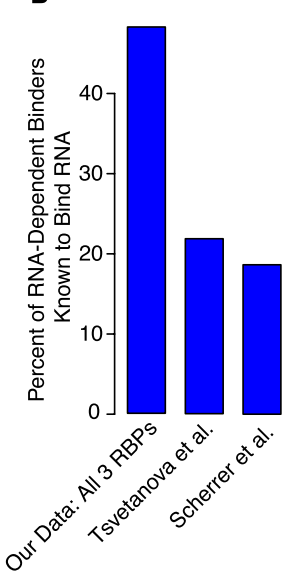

C

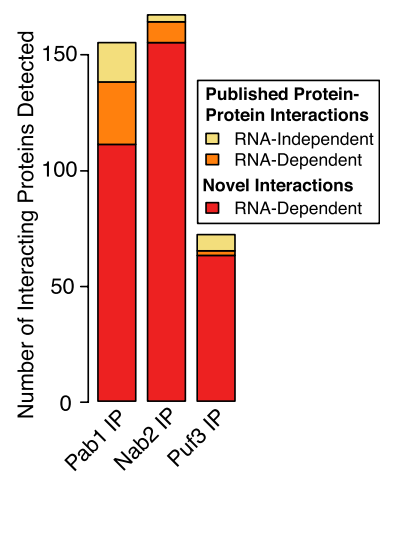

Figure 3. Barplots showing enrichment of known RNA-binding proteins and the RNA dependence of published protein-protein interactions. $(A)$ The fraction of proteins we identified as RNA-dependent binders that are known RBPs (defined as those that have domains known to bind RNA or have a molecular function of RNA binding in the Gene Ontology database). Known RNA-binding proteins are significantly enriched among the proteins interacting in an RNA-dependent manner with Pab1, Nab2 and Puf3 (for example, $P$-value of $2 \times 10^{-35}$ for the union of all three data sets). (B) The percentage of proteins known to bind RNA in the set of 220 RNA-dependent binders in our combined data set, compared with the top 220 proteins from the protein array data from two previously published attempts to identify proteome-wide RNA-protein interactions. All had significant enrichment of known RBPs, but the enrichment seen with the set of proteins identified by our method was much greater (hypergeometric $P$-values $2 \times 10^{-35}, 2 \times 10^{-4}$, and $2 \times 10^{-5}$ for this study, Scherrer et al. 2010, and Tsvetanova et al. 2010, respectively). (C) A barplot showing the RNA dependence of published high-confidence protein-protein interactions and also the number of novel interactions (RNA dependent) we observed with Pab1, Nab2, and Puf3.

our data and that from either protein array data set. These results suggest that our method both corroborates and extends previous work identifying RNA-interacting proteins.

We analyzed the enrichment of Gene Ontology (GO) terms, protein domains from the protein families database (PFAM), and biological pathways from the Kyoto Encyclopedia of Genes and Genomes (KEGG) relative to all the proteins that could be detected from an analysis of a yeast whole-cell lysate (Fig. 4). Our data clearly partition the proteins into groups with strong ties to RNA-dependent or independent binding as evidenced by the enrichment of GO terms, PFAM domains, and KEGG pathways. The proteins we classified as having RNA-dependent interactions with Pab1, Nab2, or Puf3 were enriched for Gene Ontology (GO) terms referring to RNA-related biological processes and molecular functions, such as RNA binding, transcription, splicing, translation, and decay (Fig. 4). Importantly, the majority of these RNArelated GO terms were not similarly enriched among the proteins falling below the threshold we set for classification as RNAdependent binders, again demonstrating that our method had successfully separated these proteins based on their ability to bind RNA. The proteins with RNA-dependent interactions were also enriched for several protein domains known to bind RNA, DNA, or nucleic acid in general, such as SWIRM nucleic acid-binding domains, LSM RNA-binding domains, RNA recognition motif domains, MIF4G protein- and nucleic acid-binding domains, La RNAbinding domains, and DEAD/DEAH-box helicase domains. None of these domains were enriched among the proteins falling below the cutoff for RNA-dependent interactions.

Kyoto Encyclopedia of Genes and Genomes (KEGG) pathway enrichment provides further insight into the functional roles of Pab1, Nab2, and Puf3. Specifically, the proteins that interact in an RNA-dependent manner with Pab1 and Nab2, but not those that known role of Puf3 in promoting the degradation of its mRNA targets (Gerber et al. 2004; Lee et al. 2010).

\section{Identification and validation of novel RNA-binding proteins}

The strong enrichment of known RNA-binding proteins that we observed among the 220 RNA-dependent binders (Fig. 3A) makes it likely that most of the 114 proteins in this group that are not currently annotated as RNA-binding proteins also bind RNA (Supplemental Fig. S6; Supplemental Table S5). To test whether these candidate RBPs bind directly to RNA, we used a method based in part on previous approaches (Greenberg 1979, 1980; Ule et al. 2005) that combines UV cross-linking, affinity purification, RNase treatment, polynucleotide kinase labeling with ${ }^{32} \mathrm{P}$, and denaturing SDS-PAGE electrophoresis (Supplemental Fig. S7). This method allows us to identify whether a candidate RBP makes direct contact with RNA (within 1 A) (Pramanik and Bewley 1996; Ule et al. 2005). We tested 25 of the 76 candidate RBPs that were not reported to interact physically with known RBPs as well as five of the 38 candidates that have been reported to interact physically with known RNA-binding proteins. We also included 10 known RBPs as positive controls and five putative negative control proteins that were selected from among highly abundant proteins (95th percentile for abundance) for which we had no evidence to suggest that they bind RNA.

We quantified any detectable radioactive bands on our denaturing SDS-PAGE gels corresponding to the candidate RBPs and analyzed the relationship between the molecules of cross-linked RNA that were detected and an estimate of the molecules of each protein present (based on data from the Saccharomyces Genome Database [Ghaemmaghami et al. 2003; de Godoy et al. 2008; Cherry et al. 2012] and described in Methods). This revealed a correlation 


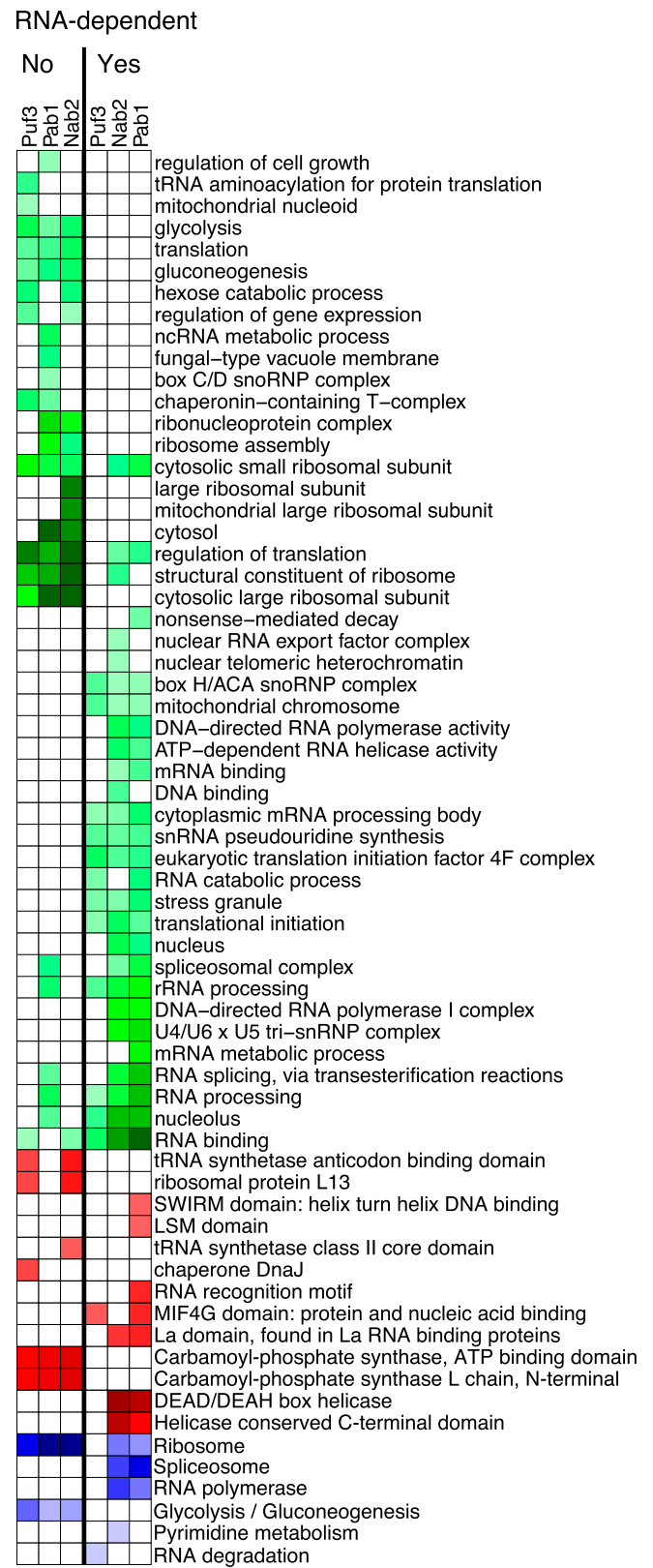

cules of RNA than would be expected based on their high abundance, placing them in the bottom $10 \%$ of cross-linking efficiency out of all 45 tested proteins (Table 1).

We used the cross-linking efficiency of the negative controls to set a threshold for validated RNA-binding proteins. There were 23 candidate RBPs above this threshold (out of the 25 with detectable cross-linking); their cross-linking efficiencies ranged from $\sim 0.1$ to $0.001 \%$. Remarkably, the four proteins that crosslinked with the highest efficiency among all 45 proteins we tested, including 10 known RBPs, were newly identified candidate RBPs (Table 1). We also found that whether or not a candidate RBP had been reported to interact physically with a known RBP was not a strong predictor of whether it could be cross-linked directly to RNA in our assay ( $4 / 5$ for the potential indirect binders and 19/25 for the others). Overall, $\sim 77 \%$ (23 of 30 ) of the candidate RBPs cross-linked to RNA with higher efficiency than the negative controls, providing strong evidence that the majority of the novel candidate RBPs discovered in this study may bind directly to RNA.

None of these 23 validated novel RBPs have any known RNA-binding domains. While many of these proteins have unidentified molecular functions, those with known roles and pathways are remarkably diverse, including a vesicle trafficking protein (Sec16), a transcription factor (Mbf1), a DNA-binding protein (Stm1), two helicases (Ecm32, Slh1), a metabolic enzyme (Imd4), a GTPase (Vps1), and a histone acetyltransferase (Eaf3) (Table 1). The unsuspected RNAbinding activity of dozens of proteins found here underscores the need for unbiased methods for discovering novel RNA-binding proteins.

Figure 4. Differential enrichment of Gene Ontology terms, PFAM domains, and KEGG pathways. A heatmap showing the enrichment of Gene Ontology terms, PFAM domains, and KEGG pathways among the proteins we identified as RNA-dependent binders and those that were not (labeled "Yes" and "No," respectively). Enrichment of Gene Ontology terms, PFAM domains, and KEGG pathways is depicted in green, red, and blue, respectively. Colors correspond to the negative log base 10 of the hypergeometric $P$-values. The columns are enrichment seen among proteins interacting in an RNAdependent manner with Puf3, Pab1, or Nab2.

between protein abundance and molecules of RNA cross-linked (Pearson correlation coefficient of 0.8 for known RBPs and 0.7 for all proteins), although there was a large variation in the amount of RNA that could be cross-linked for proteins at the same abundance level. For example, the known RBPs She3 and Puf3 have similar protein abundance $(\sim 1000$ and $\sim 850$ molecules per cell, respectively), but Puf3 cross-links to $\sim 40$-fold more molecules of RNA than She3. For more discussion of these differences in crosslinking efficiency, see the Supplemental Material. The two negative controls with detectable bands cross-linked to far fewer moledomains known to bind RNA, we sought to identify the region of each protein that cross-links to RNA. We performed a partial protease digest of purified proteins to reveal structured regions cross-linked to radioactively labeled, exhaustively digested RNA fragments. We then analyzed the digestion products by SDS-PAGE to find distinct bands representing ordered domains of the proof each of these bands to determine if they were cross-linked to RNA. Protease digestion of Mbf1 produced fragments that could be resolved as three distinct bands by SDS-PAGE, corresponding tein (Fontana et al. 2012). Next, we measured the radioactivity

\section{Genome Research www.genome.org}


Table 1. Results of UV-cross-linking assay

\begin{tabular}{|c|c|c|c|c|c|}
\hline$\underline{\text { Rank }}$ & Protein & \% X-linked & $\begin{array}{l}\text { RNA Binding } \\
\text { Domain }\end{array}$ & Molecular Function & Description \\
\hline 1 & Ylr419w & $0.15 \%$ & unknown & unknown & Putative helicase with limited sequence similarity to human $\mathrm{Rb}$ protein \\
\hline 2 & Sec16 & $0.14 \%$ & unknown & protein anchor & COPII vesicle coat protein required for ER transport vesicle budding \\
\hline 3 & Pat1 & $0.10 \%$ & unknown & unknown & Topoisomerase II-associated deadenylation-dependent mRNA-decapping factor \\
\hline 4 & Psp2 & $0.09 \%$ & unknown & unknown & High-copy suppressor of group II intron-splicing defects \\
\hline 5 & Puf3 & $0.08 \%$ & Pumilio & mRNA binding & mitochondrial, promotes degradation of nuclear-encoded mitochondrial mRNAs \\
\hline 6 & Hrb1 & $0.07 \%$ & RRM & RNA binding & involved in the export of mRNAs from the nucleus to the cytoplasm \\
\hline 7 & Bem2 & $0.05 \%$ & unknown & Rho GTPase activator & involved in the control of cytoskeleton organization and cellular morphogenesis \\
\hline 8 & Nab2 & $0.04 \%$ & unknown & poly(A) RNA binding & required for nuclear mRNA export and poly(A) tail length control \\
\hline 9 & Ecm32 & $0.04 \%$ & unknown & DNA/RNA helicase & DNA dependent ATPase/DNA helicase involved in modulating translation termination \\
\hline 10 & Clu1 & $0.03 \%$ & unknown & unknown & deletion causes mitochondrial defects but none in growth or translation initiation \\
\hline 11 & Gbp2 & $0.02 \%$ & RRM & RNA binding & involved in the export of mRNAs from the nucleus to the cytoplasm \\
\hline 12 & Pub1 & $0.02 \%$ & RRM & mRNA/poly(U) binding & abundant mRNP-component that is required for stability of many mRNAs \\
\hline 13 & Pab1 & $0.02 \%$ & RRM & poly(A) RNA binding & part of $3^{\prime}$-end RNA-processing, mediates interactions between the $5^{\prime}$ cap and the $3^{\prime}$ tail \\
\hline 14 & Rmd9 & $0.02 \%$ & unknown & unknown & possible role in delivering mitochondrial mRNAs to ribosomes \\
\hline 15 & $\operatorname{Sln} 1$ & $0.02 \%$ & unknown & Putative RNA helicase & related to Ski2p, involved in translation inhibition of non-poly $(A)$ mRNAs \\
\hline 16 & Ydr348c & $0.01 \%$ & unknown & unknown & Protein of unknown function thought to be involved in endocytosis \\
\hline 17 & Svl3 & $0.01 \%$ & unknown & unknown & Protein of unknown function, mutant phenotype suggests a role in vacuolar function \\
\hline 18 & Imd4 & $0.009 \%$ & unknown & IMP dehydrogenase & catalyzes the first step of GMP biosynthesis \\
\hline 19 & Stm1 & $0.007 \%$ & unknown & DNA binding & protein required for optimal translation under nutrient stress \\
\hline 20 & Mbf1 & $0.007 \%$ & unknown & transcription coactivator & bridges the DNA-binding region of Gcn4p and TATA-binding protein Spt15p \\
\hline 21 & Scd6 & $0.006 \%$ & Sm-like & RNA binding & may have a role in RNA processing \\
\hline 22 & Nup1 & $0.005 \%$ & unknown & structural molecule activity & Nuclear pore complex (NPC), involved in protein import/export and in export of RNAs \\
\hline 23 & Pin4 & $0.002 \%$ & RRM & unknown & Protein involved in G2/M phase progression and response to DNA damage \\
\hline 24 & She3 & $0.002 \%$ & unknown & mRNA binding & part of the mRNA localization machinery for certain bud localized proteins \\
\hline 25 & Vps1 & $0.002 \%$ & unknown & GTPase & required for vacuolar sorting \\
\hline 26 & Aep1 & $0.002 \%$ & unknown & unknown & Protein required for expression of the mitochondrial subunit 9 of F1-F0 ATP synthase \\
\hline 27 & Rbg1 & $0.001 \%$ & unknown & GTP binding & member of the DRG family, associates with translating ribosomes \\
\hline 28 & Eaf3 & $0.001 \%$ & unknown & histone acetyltransferase & nonessential component of the NuA4 acetyltransferase complex, DNA repair \\
\hline 29 & Syp1 & $0.001 \%$ & unknown & unknown & involved in endocytic site formation, may regulate assembly/disassembly of septin ring \\
\hline 30 & Aep2 & $0.001 \%$ & unknown & unknown & likely involved in translation of the mitochondrial subunit F1-F0 ATP synthase mRNA \\
\hline 31 & Iki1 & $0.0006 \%$ & unknown & unknown & Subunit of Elongator complex, required for modification of wobble nucleosides in tRNA \\
\hline 32 & Yhr097c & $\begin{array}{l}\text { No protein } \\
\text { quant. }\end{array}$ & unknown & unknown & Putative protein of unknown function \\
\hline 33 & Cpr1 & $0.0003 \%$ & unknown & peptidyl-prolyl isomerase & peptidyl-prolyl cis-trans isomerase \\
\hline 34 & Bat1 & $0.0002 \%$ & unknown & amino-acid transaminase & mitochondrial branched-chain amino acid (BCAA) aminotransferase \\
\hline 35 & Tom71 & $0.0001 \%$ & unknown & protein transporter & mitochondrial outer membrane protein with similarity to Tom70p \\
\hline 36 & Bmh1 & $0.00003 \%$ & unknown & DNA rep. origin binding & 14-3-3 protein, major isoform, binds proteins and DNA \\
\hline 37 & Gcd11 & No band & unknown & translation initiation factor & Gamma subunit of elF2, involved in the identification of the start codon \\
\hline 38 & Cin8 & No band & unknown & $(+/-)$ end microtubule motor & Kinesin motor protein involved in mitotic spindle assembly and chromosome segregation \\
\hline 39 & Ppt1 & No band & unknown & protein Ser/Thr phosphatase & nucleus and cytoplasm, potential role in phosphate metabolism and rRNA processing \\
\hline 40 & Tfa2 & No band & unknown & ssDNA binding & TFIIE small subunit, involved in RNA polymerase II transcription initiation \\
\hline 41 & Vam6 & No band & unknown & Rab guanyl exchange factor & Vacuolar protein that plays a critical role in the tethering for vacuolar membrane fusion \\
\hline 42 & Mip6 & No band & RRM & RNA binding & interacts with Mex67, a component of the nuclear pore involved in mRNA export \\
\hline 43 & Ccw12 & No band & unknown & unknown & Cell wall mannoprotein, role in maintenance of newly synthesized areas of cell wall \\
\hline 44 & Erg11 & No band & unknown & sterol 14-demethylase & lanosterol 14-alpha-demethylase in the ergosterol biosynthesis pathway \\
\hline 45 & Lcb2 & No band & unknown & palmitoyltransferase & responsible for the first committed step in sphingolipid synthesis \\
\hline
\end{tabular}

(Green) The known RBPs; (red) negative controls; (white) candidate RBPs. Cross-linking efficiency was calculated by comparing the molecules of crosslinked RNA to the molecules of protein. The proteins that had detectable bands in our UV-cross-linking assay are ranked by cross-linking efficiency. (Horizontal black line) The empirical cutoff we drew based on the highest cross-linking efficiency observed among the negative control proteins. There were 23 candidate RBPs above this cutoff. Note that while Yhr097c has an unknown protein abundance, it cross-linked to a similar number of molecules of RNA as the negative controls (which are above the 95th percentile for protein abundance). Therefore, there is a $>95 \%$ chance that Yhr097c cross-links with higher efficiency than the negative controls.

to putative stable digestion products (Fig. 5A). Bands 2 and 3 had strong signals from the cross-linked, radiolabeled RNA, while band 1 did not (Fig. 5A). We excised these three bands (and undigested Mbf1) from the gel and analyzed them by mass spectrometry, comparing the enrichment of each peptide relative to undigested Mbf1 for each band after normalization (Fig. 5B). This identified a region at the $\mathrm{N}$ terminus in the multiprotein bridging factor (MBF) domain that was $\sim 10$-fold enriched in bands 2 and 3 but not band 1 (Fig. 5B). Conversely, band 1, which did not crosslink to RNA, displayed approximately twofold enrichment for peptides derived from the helix-turn-helix DNA-binding domain. These results imply that the RNA-binding domain of Mbf1 is distinct from its DNA-binding domain, suggesting that Mbf1 could potentially bind simultaneously to DNA and RNA.

A large fraction of the RNA-dependent binders that we identified are annotated as DNA-binding proteins, including several transcription factors such as Mbf1 (Supplemental Fig. S6). We speculate that the RNA-dependent binders that also bind DNA may operate to connect the post-transcriptional regulatory network to the transcriptional regulatory network, by first binding
DNA to regulate transcription and subsequently binding to the nascent RNA to affect its stability or translation in the cytoplasm. Indeed, recent reports provide evidence that transcriptional regulation can affect post-transcriptional regulation in yeast (HarelSharvit et al. 2010; Bregman et al. 2011; Choder 2011). In addition, a connection between the transcription and the processing of RNA has long been known to exist (Cramer et al. 1997; McCracken et al. 1997).

\section{Analysis of the proteins that bind to RNAs concurrently with Nab2 or Puf 3 expands on the existing models of Nab2 and Puf 3 function in post-transcriptional regulation}

While RNA immunoprecipitation methods (RIP-chip, CLIP-seq, and related) can identify specific interactions between RNAs and RNA-binding proteins, they cannot identify whether the multiple proteins that interact with a given RNA bind concurrently, sequentially, or in mutually exclusive cellular locations. In contrast, our RNA-dependent interaction data enable us to directly identify pairs of proteins that bind concurrently to one or more RNAs in 
A

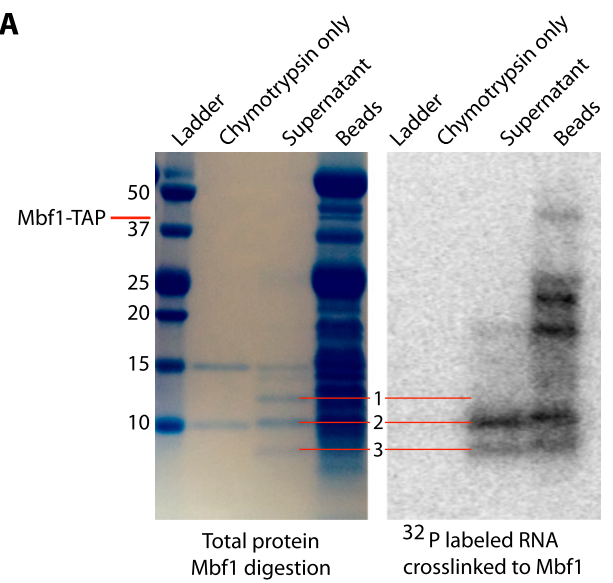

B

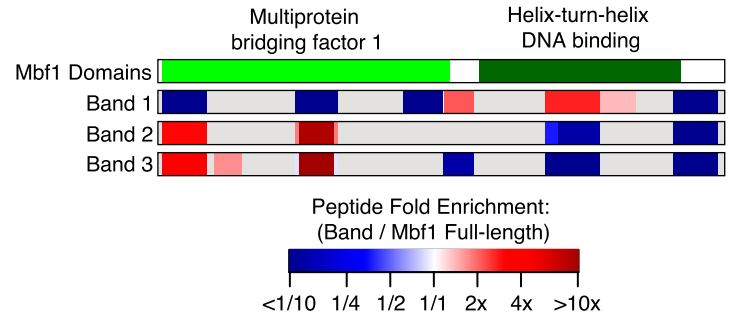

Figure 5. Mapping the RNA-binding domain of Mbf1. (A) SDS-PAGE analysis of protein fragments resulting from partial digestion of Mbf1 with the protease chymotrypsin. The lanes from left to right contain the ladder, chymotrypsin only, the supernatant of protein fragments liberated by chymotrypsin digestion of Mbf1, and the protein fragments remaining on the beads. A total protein stain is shown on the left, and the radioactive image of the same gel is shown on the right. Gel images were scaled and aligned to facilitate direct comparison of the visible bands. The radioactive image shows a signal from ${ }^{32} \mathrm{P}$-labeled RNA fragments cross-linked to Mbf1. (B) A diagram showing the domains of Mbf1 and the position and enrichment relative to full-length Mbf1 for all the peptides that were detected in each sample. The fold enrichment of the normalized intensity of each peptide relative to undigested Mbf1 for each of the three bands is represented by a color gradient ranging from dark blue for less than one-tenth fold enriched, to white for no enrichment, to dark red for greater than 10-fold enriched. (Gray) Areas of the protein for which no peptides were detected.

a cell. Together with other information about these RBPs, this can provide clues to its function and when and where in the cell it binds.

$\mathrm{Nab} 2$ is involved in the end processing, polyadenylation, and export of poly(A) mRNA from the nucleus (Green et al. 2002; Hector et al. 2002; Fasken et al. 2008; Iglesias and Stutz 2008; Tutucci and Stutz 2011). It is generally believed that Nab2 binds to mRNAs during their end cleavage and polyadenylation and is removed immediately following their nuclear export (Lee and Aitchison 1999; Tran et al. 2007). Previous work also revealed that the mRNAs bound by Nab2 tend to encode nuclear-localized proteins involved in transcription and splicing (Guisbert et al. 2005; Hogan et al. 2008). We confirmed several known RNA-dependent interactions with Nab2 in our data and uncovered novel RNAdependent interactions that were consistent with the known role of Nab2 in end processing and mRNA export, such as THO/TREX complex components, Mex67, Mtr2, and Nup1 (Fig. 6).

Using our data to extend the existing model of Nab2 function, we looked for novel RNA-dependent interactions between $\mathrm{Nab} 2$ and other well-studied RNA-binding proteins involved in processes other than mRNA polyadenylation and export (Fig. 6). We detected novel RNA-dependent interactions between Nab2 and several protein components of the splicing apparatus (Smb1, Smd1, Smd2, Smd3, Smx3, Cef1, Luc7, Ms15, Prp19, Prp22, Prp39, and Yhc1) (Fig. 6). We also found RNA-dependent interactions with proteins involved in transcription or the regulation of transcription (Tfa2, Arp9, Gat1, Mbf1, Met28, and the RNA polymerase II central core component Rpb2) (Fig. 6). These interactions appear to be specific to Nab2, because most are not seen with Pab1 $(5 / 5 \mathrm{Sm}$ proteins, $1 / 7$ other splicing, $1 / 6$ transcription related) or Puf3 (0 out of 18). Nab2's unexpected RNA-dependent interactions with these proteins involved in splicing and transcription suggest that in some cases Nab2 may bind earlier than generally believed, perhaps cotranscriptionally. We also find a novel RNA-dependent interaction between Nab2 and the nuclear exosome core component Rrp6, suggesting that Nab2 remains associated with some mRNAs when they are targeted for surveillance or degradation. Finally, while in vitro experiments and genetic interactions have led to the model that Nab2 is removed from its mRNA targets by helicases anchored on the cytoplasmic face of the nuclear pore complex (Tran et al. 2007), we discovered novel RNA-dependent interactions between $\mathrm{Nab} 2$ and proteins involved in translation and the repression of translation, such as Tif4631, Tif4632, Cdc33, Sbp1, Khd1, and Pab1. This suggests that in some cases Nab2 remains bound to its targets after mRNA export (Fig. 6). These results illustrate how analyzing the well-studied RBPs that bind concurrently with Nab2 can expand the model of Nab2 function and refine our view of when in the life of its mRNA targets it binds.

Applying a similar approach to Puf3 identifies several novel RNA-dependent interactions that extend and refine the known role of Puf3 in repressing the expression of its mRNA targets. Puf3 promotes the decay and localization of its mRNA targets (Gerber et al. 2004; Saint-Georges et al. 2008; Lee et al. 2010; Quenault et al. 2011). It also physically interacts with decay proteins such as the major cytoplasmic deadenylase complex Ccr4-Not (in an RNAindependent manner) (Lee et al. 2010). Our method has revealed that in addition to its role in promoting decay and localization, Puf3 binds to mRNAs concurrently with proteins involved in translation and translational repression, namely, Tif4631, Tif4632, Cdc33, Pat1, and Stm1 (Fig. 7). We have also discovered novel RNA-dependent interactions between Puf3 and the P-body and RNA decay-related proteins, Xrn1 and the Lsm ring complex (Fig. 7). Finally, we learned that Puf3 can bind to mRNAs concurrently with the stress granule proteins Sgn1 and Pub1 (Fig. 7). Puf3 can promote the deadenylation and decay of its mRNA targets independent of Ccr4 (Lee et al. 2010). It has been hypothesized to recruit an as-yet-unknown factor or factors to promote the rearrangement of the mRNP structure from a pro-translation/stability state into an anti-translation/decay state (Lee et al. 2010). Given that Stm1 and the Pat1/Lsm-ring complex are involved in the repression of translation and promote mRNA decapping/decay (Marnef and Standart 2010; Balagopal and Parker 2011), we speculate that these proteins may be the undiscovered factors that Puf3 recruits to its target mRNAs to promote their degradation. Going beyond the known role of Puf3, we found novel RNAdependent interactions between Puf3 and proteins involved in repressing translation, suggesting that Puf3 may also repress the expression of its mRNA targets at the translational level. These vignettes illustrate how our data provide a unique perspective into the makeup of the RNA-protein complexes in which an RBP of interest is found and highlight the value of this technique for the study of post-transcriptional regulation. 


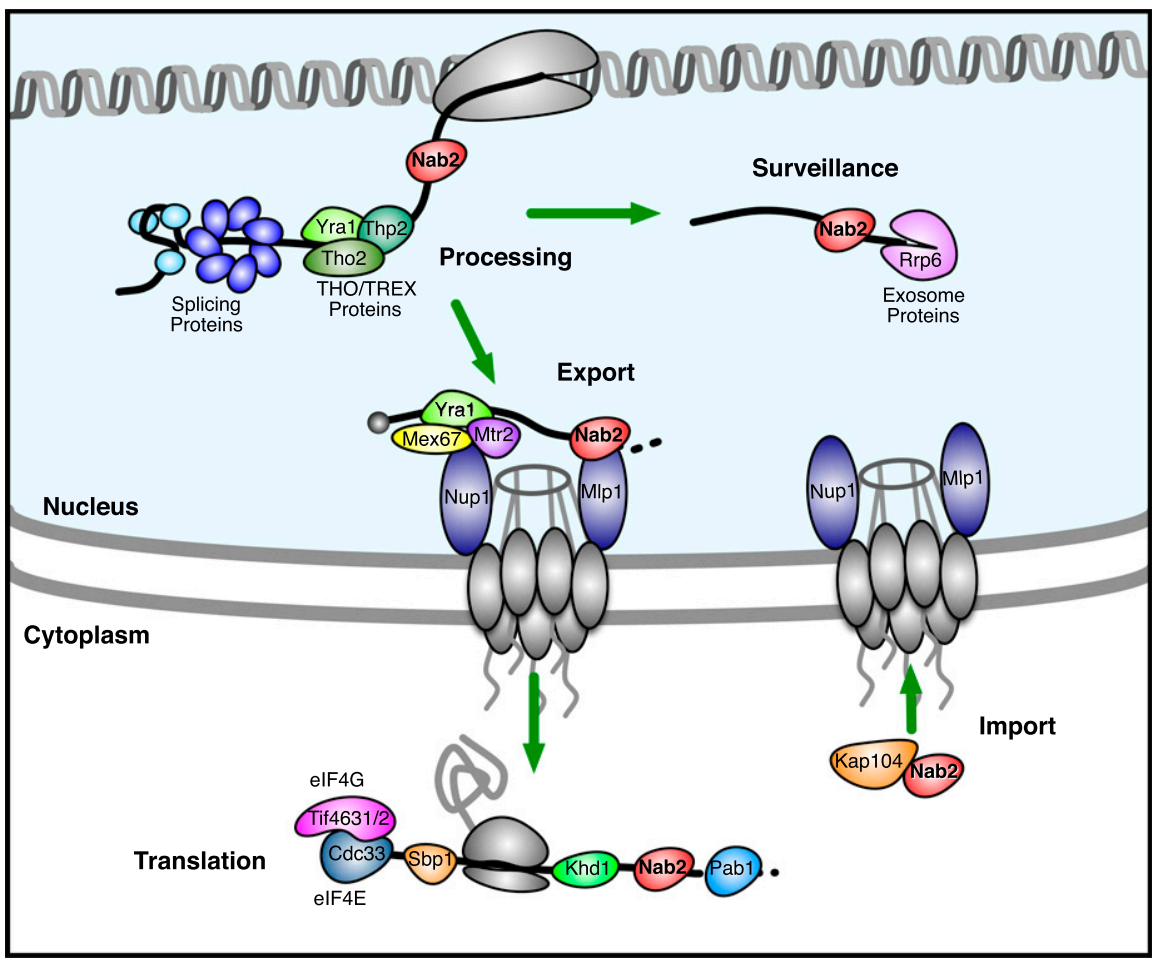

Figure 6. A revised model for Nab2 activity. This diagram depicts a subset of the proteins that we found to interact with Nab2 in an RNA-dependent or independent manner. We used our observations to expand on previous models of Nab2 function. Proteins separated by a black line (the RNA) with Nab2 had RNA-dependent interactions. When limited to well-studied proteins known to bind RNA, this RNA-dependent interaction data suggest that these RBPs bind to the same RNAs at the same time as Nab2. Proteins were placed in this diagram according to their known roles in RNA processing and regulation. Note that the RNA-independent interactions we detected between Nab2 and Mlp1 and Kap104 are also shown because of their known roles in Nab2 function.

\section{Discussion}

A growing body of evidence suggests that post-transcriptional regulation mediated by RBPs is a widespread phenomenon, but how this happens largely remains to be discovered. It is clear from the many published examples of regulatory RNA-binding activity in unexpected proteins that we need methods to enable the unbiased discovery of novel RNA-protein interactions. A prevailing model of post-transcriptional regulation is that the specific complement of RBPs bound to a given mRNA specifies its posttranscriptional fate-yet nearly all existing data are limited to defining pairwise interactions between a single RBP and a single mRNA species, potentially missing vital information about this aspect of post-transcriptional regulation.

Here we developed a method that characterizes RNA-protein interactions from a different perspective. It combines quantitative mass spectrometry with RNase treatment of affinity-purified RNA-protein complexes assembled in vivo. We interrogated the constituents of RNA-protein complexes containing the known RNA-binding proteins Pab1, Nab2, or Puf3, respectively, providing a new perspective on the role of Nab2 and Puf3 in posttranscriptional regulation.

Our data revealed a large and diverse group of previously unrecognized RNA-binding proteins and showed that the majority of previously reported protein-protein interactions involving Pab1 or Nab2 that we could detect are, in fact, RNA dependent. We extrapolate that other reported protein-protein interactions, especially those involving abundant RNAbinding proteins, may likewise reflect concurrent binding to RNA rather than direct interactions. We identified several annotated DNA-binding proteins as RNA-dependent binders. These proteins may both bind DNA to regulate transcription and subsequently bind to the nascent RNA and regulate its stability or translation in the cytoplasm, as a means of coordinating the transcriptional and post-transcriptional regulation of a given gene, a model that has been suggested by previous work (Cramer et al. 1997; McCracken et al. 1997; HarelSharvit et al. 2010; Bregman et al. 2011). In contrast to previous applications of mass spectrometry to the identification of RNA-protein interactions, our approach appears to be largely unbiased by protein abundance. Strikingly, 50\% (114/220) of the RNA-dependent binders we identified were already known to be RBPs (enrichment $P$-value $2 \times 10^{-35}$ ), which is a considerable improvement over previous approaches.

The RBP Nab2 is involved in the end processing, polyadenylation, and export of poly(A) mRNA from the nucleus; we see both known and novel RNAdependent interactions with Nab2 that are consistent with the existing model (Hector et al. 2002; Tran et al. 2007; Fasken et al. 2008; Iglesias and Stutz 2008; Tutucci and Stutz 2011). However, our data provide new insight into the temporal program of Nab2 binding based on evidence for concurrent binding with RBPs involved in transcription, splicing, and translation. From its RNA-dependent interaction partners, we infer a model in which Nab2 binds cotranscriptionally and remains bound during splicing and end

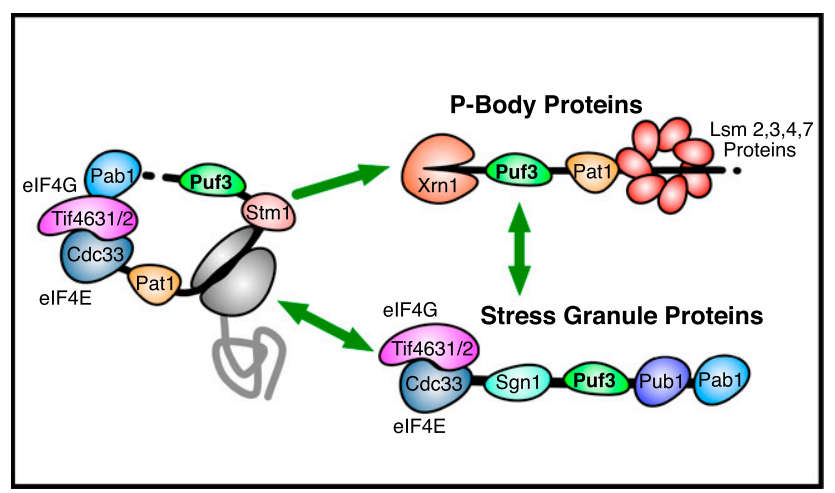

Figure 7. Insights from RNA-dependent interactions with Puf3. This diagram depicts a subset of the proteins that we found to interact with Puf 3 in an RNA-dependent manner. Proteins separated by a black line (the RNA) with Puf3 had RNA-dependent interactions. When limited to wellstudied proteins known to bind RNA, these RNA-dependent interaction data suggest that these RBPs bind to the same RNAs at the same time as Puf3. Proteins were placed in this diagram according to their known roles in RNA regulation. 
processing. Nab2 also appears to remain bound to mRNAs that fail splicing or are otherwise targeted for surveillance/degradation by the nuclear exosome. For mRNAs that pass nuclear quality control, $\mathrm{Nab} 2$ interacts with the nuclear pore to promote their export. After export into the cytoplasm, Nab2 remains bound to its mRNA targets as they are bound by cytoplasmic translation regulatory proteins and perhaps until they initiate the first round of translation. An intriguing possibility is that some of these proteins typically involved in regulating translation in the cytoplasm may be loaded onto mRNAs before or during export, while Nab2 is still bound.

The RBP Puf3 is known to promote the decay of its mRNA targets (Gerber et al. 2004; Saint-Georges et al. 2008; Lee et al. 2010; Quenault et al. 2011). We identified novel RNA-dependent interactions consistent with this role. Proteins that bind RNAs concurrently with Puf3 include candidates (Stm1 and the Pat1/Lsm1 ring complex) for hypothetical factors recruited by Puf3 to promote the rearrangement of its mRNP structure from a pro-translation/ stability state into an anti-translation/decay state (Lee et al. 2010). We found that Puf3 binds to mRNAs at the same time as proteins that are involved in translational repression (Pat1, Stm1), found in P-bodies (Xrn1, Lsm ring), or found in stress granules (Sgn1, Pub1), suggesting that Puf3 may repress its mRNA targets at the translational level as well. As a part of this model, these proteins may briefly physically interact with Puf3 as they are recruited to a Puf3-bound mRNA, but we would not necessarily expect to detect this interaction in our assay if at steady state a substantial fraction of these proteins remain bound to RNA but not Puf3.

We identified as candidate RBPs 106 proteins that were not previously known to bind RNA. Of the 30 candidates we tested, $23(77 \%)$ bound directly to RNA in an independent assay. None of these 23 novel RNA-binding proteins have known RNA-binding domains, and many have unknown molecular functions. The known functions of the novel RBPs were diverse, including a vesicle trafficking protein (Sec16), a transcriptional coactivator (Mbf1), a regulator of translational elongation (Stm1), two helicases (Ecm32 and Slh1), a metabolic enzyme (Imd4), a dynamin-like GTPase (Vps1), and a histone acetyltransferase (Eaf3). We speculate that in some cases these unexpected RNA-protein interactions involving proteins with already established biological functions that are apparently unrelated to RNA binding might have evolved to facilitate mRNA localization. Specifically, RNAs may have evolved structured elements to bind to specific proteins with distinct localization patterns (such as Imd4) to "hitch a ride" to, or hold their position in, a particular part of the cell. Overall, our high success rate for validating the RNA-binding activity of the proteins identified as RDBs suggests that many of the 76 candidates we have yet to test may also bind directly to RNA (Supplemental Table S5).

Existing methods that use microarrays or sequencing to identify the RNA targets of specific RBPs can identify sets of RBPs that interact with common RNA targets. The method we describe here makes it possible to determine which of those interactions are concurrent. This is a crucial distinction, because while each RNA may be bound by several different RBPs over the course of its lifetime (Hogan et al. 2008), many of those RBPs may bind at different times or places within the cell. When applied to a specific RNA-binding protein, the identity of other concurrently associated RBPs can provide clues to its position in the temporal sequence of protein-RNA interactions and the subcellular location in which they occur. This approach could thus be broadly applicable to mapping relationships and connections in the RNA-protein network that affects the fate of each mRNA. A similar approach could also be used to identify proteins that bind to DNA concurrently.

\section{Methods}

\section{RNA-dependent protein purification}

We grew TAP-tagged yeast strains (Pab1-TAP, Nab2-TAP, and Puf3TAP) auxotrophic for lysine to mid-log phase in media with or without heavy labeled L-lysine. We lysed the cells and purified the RNA-binding proteins essentially as described previously (Tsvetanova et al. 2010), except that we split the beads equally after the initial washes and performed the subsequent three washes in buffer with or without RNase (in excess). Note: For the Pab1 $\mathrm{Mg}^{2+}$ purification, the wash buffers contained $1.8 \mathrm{mM} \mathrm{MgCl}_{2}$, but for all other purifications, the washes were done with buffer containing $10 \mathrm{mM}$ EDTA. Finally, we boiled the beads in Laemmli sample buffer and proceeded to analysis by mass spectrometry. A detailed protocol is available in the Supplemental Material.

\section{Quantitative mass spectrometry}

Proteins were separated by SDS-PAGE, and each lane was sliced into eight fractions, which were further minced. The minced gel pieces were then destained, minced, alkylated, and incubated overnight with LysC. The resulting peptides were then extracted from the gel, separated by capillary chromatography, and analyzed by an LTQ-Orbitrap XL. The MS data were processed using the MaxQuant software suite (version 1.2.0.18) (Cox and Mann 2008) and a yeast protein database (6717 entries and its reverse complement). For the search, oxidation on methionine and protein $\mathrm{N}$-terminal acetylation were set as variable modifications. Protease cleavage specificity was set to LysC. False discovery rates at the peptide and protein levels were set to 0.01 , and only proteins with at least two quantitation events were considered for the subsequent bioinformatic analysis. A detailed protocol is available in the Supplemental Material.

\section{Analysis of mass spectrometry data}

The forward experiment (heavy labeled without RNase over unlabeled with RNase) and the reverse experiment (heavy labeled with RNase over unlabeled without RNase) were analyzed by mass spectrometry separately and treated as replicates (except with inverted heavy-to-light ratios). To generate a high-confidence data set, we filtered the mass spectrometry results for proteins for which we detected two peptides in both the forward and the reverse experiment (that map to only one protein). To generate a background set of all proteins that had the opportunity to be detected in our assays, we used mass spectrometry data from an analysis of all proteins detected from a yeast whole-cell lysate and filtered for two peptides in at least two replicates. This background set (Supplemental Table S7) was used to calculate enrichment of known RNA-binding proteins as well as Gene Ontology terms, KEGG pathways, and PFAM domains. We then inverted the heavy-to-light ratios for the reverse experiment, normalized the forward and reverse samples so that the ratio for the TAPtagged protein was 1 , and then averaged the forward and reverse values. We then took the log base 2 of these heavy-to-light ratios and worked with the data in this format from this point on [referred to as $\log _{2}(-/+)$ RNase ratios or RNA-dependence values].

To establish a conservative cutoff for the classification of proteins into RNA-dependent binders and protein binders based on their RNA-dependence values, we modeled the distributions of RNA-dependence values for proteins with RNA-independent interactions with Pab1, Nab2, and Puf3. To do this, we made two assumptions: first, that after normalization, any RNA-dependence values less than zero have a true value of zero and the observed variation from zero is due to noise; and, second, that this noise 
is symmetric about zero. Using these two assumptions, we took the RNA-dependence values less than zero (excluding the most negative $1 \%$ as extreme outliers) and combined them with their absolute values to form a null distribution symmetric about zero (Supplemental Fig. S3). We used this null distribution to determine an empirical FDR cutoff of $10 \%$ for the classification of RDBs (Supplemental Fig. S3). To evaluate this cutoff independently, we plotted the frequency of annotated RBPs in a sliding window versus the RNA-dependence values (Supplemental Fig. S4). This analysis revealed that the frequency of annotated RBPs was well above the median frequency for all proteins that could be detected from a yeast whole-cell lysate as well as the median frequency for all proteins detected in each purification experiment (Supplemental Fig. S4). Note that ribosomal proteins were excluded from the analysis for Supplemental Figures S1 and S4 because they are common mass spectrometry contaminants, and they also often have an annotated molecular function of RNA binding. This serves as independent validation of the cutoff we made for classifying proteins as RDBs.

We calculated the enrichment of Gene Ontology terms, PFAM domains, and KEGG pathways using the GOStats package in $\mathrm{R}$ (Falcon and Gentleman 2007). We corrected the resulting $P$-values for multiple hypothesis testing using the Bonferroni correction. We made the RNA-protein interaction network diagram with the program Cytoscape (Smoot et al. 2011). We made the diagrams depicting the RNA-dependent binding interactions as well as the method overview with the program OmniGraffle by the Omni Group. The protein abundance data we used were previously published (Ghaemmaghami et al. 2003). The set of high-confidence protein-protein interactions we used was from Collins et al. (2007).

\section{UV cross-linking assay}

To test whether our candidate RNA-binding proteins cross-link directly to RNA by UV irradiation, we used a method based on work by Greenberg $(1979,1980)$ and Ule et al. (2005). First, we crosslinked RNA to protein in vivo by UV irradiation and purified the TAP-tagged candidate RBPs under denaturing conditions. Then, we subjected each sample to limited digestion by MNase and then subjected half to further, exhaustive digestion by RNase. We next labeled the RNA fragments by polynucleotide kinase treatment and ran the samples on a denaturing SDS-PAGE gel. We looked specifically for the presence of a PNK-labeled band that was RNase sensitive and of corresponding size to the protein of interest. We also included 10 known RBPs as positive controls and five putative negative-control proteins that were selected from among highly abundant proteins (95th percentile for abundance) for which we had no evidence to suggest that they bind RNA. Molecules of cross-linked RNA were quantified by comparing the intensity of the radioactive band with a standard curve, and molecules of protein were estimated based on published protein abundance data and the number of cells used, the typical lysis efficiency, and the typical purification efficiency. A detailed protocol is available in the Supplemental Material.

\section{Identification of RNA-binding protein domains}

We prepared the protein samples exactly as they were for the UV-cross-linking assay described above, except that we scaled up everything $4 \times$. After we subjected the samples to exhaustive RNase digestion and radioactive labeling, we digested them with chymotrypsin, trypsin, or elastase ranging in concentration from $0.1 \mathrm{mg} / \mathrm{mL}$ to $0.0001 \mathrm{mg} / \mathrm{mL}(10 \times$ dilutions). We analyzed the supernatants containing protein fragments liberated by protease digestion by SDS-PAGE, visualizing both total protein and radioactive signal in the same gel. We analyzed the distinct bands by mass spectrometry, to map them to a specific position in the full-length protein. This information, combined with our observation of which bands were radioactively labeled, allowed us to identify the regions of the protein that were cross-linked to RNA. A detailed protocol is available in the Supplemental Material.

\section{Data access}

Raw data are included as Supplemental Material with this manuscript.

\section{Acknowledgments}

We gratefully acknowledge Alex Lovejoy and Dan Herschlag for comments on this manuscript. P.O.B., D.M.K., and G.J.H. are supported mainly by the Howard Hughes Medical Institute and by a grant from the National Institutes of Health to P.O.B. (NIH RO1 CA77097). P.O.B. is an investigator for the Howard Hughes Medical Institute. D.M.K. was also partially supported by a National Science Foundation pre-doctoral fellowship. G.J.H. was also partially supported by a Burt and Deedee McMurtry Stanford Graduate Fellowship. Work in the Mann laboratory is supported by the Max Planck Society for the Advancement of Science. The funders had no role in study design, data collection and analysis, decision to publish, or preparation of the manuscript.

\section{References}

Balagopal V, Parker R. 2011. Stm 1 modulates translation after 80 S formation in Saccharomyces cerevisiae. RNA 17: 835-842.

Baltz AG, Munschauer M, Schwanhausser B, Vasile A, Murakawa Y, Schueler M, Youngs N, Penfold-Brown D, Drew K, Milek M, et al. 2012. The mRNA-bound proteome and its global occupancy profile on protein-coding transcripts. Mol Cell 46: 674-690.

Bertrand E, Fromont-Racine M, Pictet R, Grange T. 1993. Visualization of the interaction of a regulatory protein with RNA in vivo. Proc Natl Acad Sci 90: 3496-3500.

Bohnsack MT, Martin R, Granneman S, Ruprecht M, Schleiff E, Tollervey D. 2009. Prp43 bound at different sites on the pre-rRNA performs distinct functions in ribosome synthesis. Mol Cell 36: 583-592.

Bregman A, Avraham-Kelbert M, Barkai O, Duek L, Guterman A, Choder M. 2011. Promoter elements regulate cytoplasmic mRNA decay. Cell 147: 1473-1483.

Brune C, Munchel SE, Fischer N, Podtelejnikov AV, Weis K. 2005. Yeast poly(A)-binding protein Pab1 shuttles between the nucleus and the cytoplasm and functions in mRNA export. RNA 11: 517-531.

Butter F, Scheibe M, Morl M, Mann M. 2009. Unbiased RNA-protein interaction screen by quantitative proteomics. Proc Natl Acad Sci 106: 10626-10631.

Casey JL, Hentze MW, Koeller DM, Caughman SW, Rouault TA, Klausner RD, Harford JB. 1988. Iron-responsive elements: Regulatory RNA sequences that control mRNA levels and translation. Science 240: 924-928.

Castello A, Fischer B, Eichelbaum K, Horos R, Beckmann BM, Strein C, Davey NE, Humphreys DT, Preiss T, Steinmetz LM, et al. 2012. Insights into RNA biology from an atlas of mammalian mRNA-binding proteins. Cell 149: 1393-1406.

Cherry JM, Hong EL, Amundsen C, Balakrishnan R, Binkley G, Chan ET, Christie KR, Costanzo MC, Dwight SS, Engel SR, et al. 2012. Saccharomyces Genome Database: The genomics resource of budding yeast. Nucleic Acids Res 40: D700-D705.

Choder M. 2011. mRNA imprinting: Additional level in the regulation of gene expression. Cell Logist 1: 37-40.

Collins SR, Kemmeren P, Zhao XC, Greenblatt JF, Spencer F, Holstege FC, Weissman JS, Krogan NJ. 2007. Toward a comprehensive atlas of the physical interactome of Saccharomyces cerevisiae. Mol Cell Proteomics 6: 439-450.

Cox J, Mann M. 2008. MaxQuant enables high peptide identification rates, individualized p.p.b.-range mass accuracies and proteome-wide protein quantification. Nat Biotechnol 26: 1367-1372.

Cramer P, Pesce CG, Baralle FE, Kornblihtt AR. 1997. Functional association between promoter structure and transcript alternative splicing. Proc Natl Acad Sci 94: 11456-11460. 
de Godoy LM, Olsen JV, Cox J, Nielsen ML, Hubner NC, Frohlich F, Walther TC, Mann M. 2008. Comprehensive mass-spectrometry-based proteome quantification of haploid versus diploid yeast. Nature 455: 1251-1254.

Dunn EF, Hammell CM, Hodge CA, Cole CN. 2005. Yeast poly(A)-binding protein, Pab1, and PAN, a poly(A) nuclease complex recruited by Pab1 connect mRNA biogenesis to export. Genes Dev 19: 90-103.

Falcon S, Gentleman R. 2007. Using GOstats to test gene lists for GO term association. Bioinformatics 23: 257-258.

Fasken MB, Stewart M, Corbett AH. 2008. Functional significance of the interaction between the mRNA-binding protein, Nab2, and the nuclear pore-associated protein, Mlp1, in mRNA export. J Biol Chem 283: 27130-27143.

Fontana A, de Laureto PP, Spolaore B, Frare E. 2012. Identifying disordered regions in proteins by limited proteolysis. Methods Mol Biol 896: 297-318.

Gavin AC, Aloy P, Grandi P, Krause R, Boesche M, Marzioch M, Rau C, Jensen LJ, Bastuck S, Dumpelfeld B, et al. 2006. Proteome survey reveals modularity of the yeast cell machinery. Nature 440: 631-636.

Gebauer F, Hentze MW. 2004. Molecular mechanisms of translational control. Nat Rev Mol Cell Biol 5: 827-835.

Gerber AP, Herschlag D, Brown PO. 2004. Extensive association of functionally and cytotopically related mRNAs with Puf family RNAbinding proteins in yeast. PLOS Biol 2: e79.

Ghaemmaghami S, Huh WK, Bower K, Howson RW, Belle A, Dephoure N, O'Shea EK, Weissman JS. 2003. Global analysis of protein expression in yeast. Nature 425: 737-741.

Granneman S, Kudla G, Petfalski E, Tollervey D. 2009. Identification of protein binding sites on U3 snoRNA and pre-rRNA by UV cross-linking and highthroughput analysis of cDNAs. Proc Natl Acad Sci 106: 9613-9618.

Granneman S, Petfalski E, Swiatkowska A, Tollervey D. 2010. Cracking pre$40 S$ ribosomal subunit structure by systematic analyses of RNA-protein cross-linking. EMBO J 29: 2026-2036.

Green DM, Marfatia KA, Crafton EB, Zhang X, Cheng X, Corbett AH. 2002. $\mathrm{Nab} 2 \mathrm{p}$ is required for poly(A) RNA export in Saccharomyces cerevisiae and is regulated by arginine methylation via Hmt1p. J Biol Chem 27 7: 77527760 .

Greenberg JR. 1979. Ultraviolet light-induced crosslinking of mRNA to proteins. Nucleic Acids Res 6: 715-732.

Greenberg JR. 1980. Proteins crosslinked to messenger RNA by irradiating polyribosomes with ultraviolet light. Nucleic Acids Res 8: 5685-5701.

Guisbert K, Duncan K, Li H, Guthrie C. 2005. Functional specificity of shuttling hnRNPs revealed by genome-wide analysis of their RNA binding profiles. RNA 11: 383-393.

Harel-Sharvit L, Eldad N, Haimovich G, Barkai O, Duek L, Choder M. 2010. RNA polymerase II subunits link transcription and mRNA decay to translation. Cell 143: 552-563.

Hector RE, Nykamp KR, Dheur S, Anderson JT, Non PJ, Urbinati CR, Wilson SM, Minvielle-Sebastia L, Swanson MS. 2002. Dual requirement for yeast hnRNP Nab2p in mRNA poly(A) tail length control and nuclear export. EMBO J 21: 1800-1810.

Hentze MW, Caughman SW, Rouault TA, Barriocanal JG, Dancis A, Harford JB, Klausner RD. 1987a. Identification of the iron-responsive element for the translational regulation of human ferritin mRNA. Science 238: 1570-1573.

Hentze MW, Rouault TA, Caughman SW, Dancis A, Harford JB, Klausner RD. 1987b. A cis-acting element is necessary and sufficient for translational regulation of human ferritin expression in response to iron. Proc Natl Acad Sci 84: 6730-6734.

Hieronymus H, Silver PA. 2003. Genome-wide analysis of RNA-protein interactions illustrates specificity of the mRNA export machinery. Nat Genet 33: 155-161.

Hogan DJ, Riordan DP, Gerber AP, Herschlag D, Brown PO. 2008. Diverse RNA-binding proteins interact with functionally related sets of RNAs, suggesting an extensive regulatory system. PLoS Biol 6: e255.

Hubner NC, Bird AW, Cox J, Splettstoesser B, Bandilla P, Poser I, Hyman A, Mann M. 2010. Quantitative proteomics combined with BAC TransgeneOmics reveals in vivo protein interactions. J Cell Biol 189: 739-754.

Iglesias N, Stutz F. 2008. Regulation of mRNP dynamics along the export pathway. FEBS Lett 582: 1987-1996.

Jansen JM, Wanless AG, Seidel CW, Weiss EL. 2009. Cbk1 regulation of the RNA-binding protein Ssd1 integrates cell fate with translational control. Curr Biol 19: 2114-2120.

Keene JD. 2007a. Biological clocks and the coordination theory of RNA operons and regulons. Cold Spring Harb Symp Quant Biol 72: 157-165.

Keene JD. 2007b. RNA regulons: Coordination of post-transcriptional events. Nat Rev Genet 8: 533-543.

Krogan NJ, Cagney G, Yu H, Zhong G, Guo X, Ignatchenko A, Li J, Pu S, Datta N, Tikuisis AP, et al. 2006. Global landscape of protein complexes in the yeast Saccharomyces cerevisiae. Nature 440: 637-643.

Kurischko C, Kim HK, Kuravi VK, Pratzka J, Luca FC. 2011. The yeast Cbk1 kinase regulates mRNA localization via the mRNA-binding protein Ssd1. J Cell Biol 192: 583-598.
Lee DC, Aitchison JD. 1999. Kap104p-mediated nuclear import: Nuclear localization signals in mRNA-binding proteins and the role of Ran and RNA. J Biol Chem 274: 29031-29037.

Lee D, Ohn T, Chiang YC, Quigley G, Yao G, Liu Y, Denis CL. 2010. PUF3 acceleration of deadenylation in vivo can operate independently of CCR4 activity, possibly involving effects on the PAB1-mRNP structure. J Mol Biol 399: 562-575.

Leibold EA, Munro HN. 1988. Cytoplasmic protein binds in vitro to a highly conserved sequence in the $5^{\prime}$ untranslated region of ferritin heavy-and light-subunit mRNAs. Proc Natl Acad Sci 85: 2171-2175.

Mann M. 2006. Functional and quantitative proteomics using SILAC. Nat Rev Mol Cell Biol 7: 952-958.

Marnef A, Standart N. 2010. Pat1 proteins: A life in translation, translation repression and mRNA decay. Biochem Soc Trans 38: 1602-1607.

McCracken S, Fong N, Yankulov K, Ballantyne S, Pan G, Greenblatt J, Patterson SD, Wickens M, Bentley DL. 1997. The C-terminal domain of RNA polymerase II couples mRNA processing to transcription. Nature 385: $357-361$.

Mittler G, Butter F, Mann M. 2009. A SILAC-based DNA-protein interaction screen that identifies candidate binding proteins to functional DNA elements. Genome Res 19: 284-293.

Ong SE, Mittler G, Mann M. 2004. Identifying and quantifying in vivo methylation sites by heavy methyl SILAC. Nat Methods 1: 119-126.

Paquin N, Menade M, Poirier G, Donato D, Drouet E, Chartrand P. 2007. Local activation of yeast $A S H 1$ mRNA translation through phosphorylation of Khd1p by the casein kinase Yck1p. Mol Cell 26: 795-809.

Pramanik SK, Bewley JD. 1996. Post-transcriptional regulation of protein synthesis during alfalfa embryogenesis: Proteins associated with the cytoplasmic polysomal and non-polysomal mRNAs (messenger ribonucleoprotein complex). J Exp Bot 45: 1871-1879.

Quenault T, Lithgow T, Traven A. 2011. PUF proteins: Repression, activation and mRNA localization. Trends Cell Biol 21: 104-112.

Rouault TA, Hentze MW, Haile DJ, Harford JB, Klausner RD. 1989. The ironresponsive element binding protein: A method for the affinity purification of a regulatory RNA-binding protein. Proc Natl Acad Sci 86: 5768-5772.

Saint-Georges Y, Garcia M, Delaveau T, Jourdren L, Le Crom S, Lemoine S, Tanty V, Devaux F, Jacq C. 2008. Yeast mitochondrial biogenesis: A role for the PUF RNA-binding protein Puf3p in mRNA localization. PLoS ONE 3: e2293.

Scheibe M, Butter F, Hafner M, Tuschl T, Mann M. 2012. Quantitative mass spectrometry and PAR-CLIP to identify RNA-protein interactions. Nucleic Acids Res 40: 9897-9902.

Schenk L, Meinel DM, Strasser K, Gerber AP. 2012. La-motif-dependent mRNA association with Slf1 promotes copper detoxification in yeast. RNA 18: 449-461.

Scherrer T, Mittal N, Janga SC, Gerber AP. 2010. A screen for RNA-binding proteins in yeast indicates dual functions for many enzymes. PLOS ONE 5: e15499.

Scherrer T, Femmer C, Schiess R, Aebersold R, Gerber AP. 2011. Defining potentially conserved RNA regulons of homologous zinc-finger RNAbinding proteins. Genome Biol 12: R3.

Schulze WX, Mann M. 2004. A novel proteomic screen for peptide-protein interactions. J Biol Chem 279: 10756-10764.

Smoot ME, Ono K, Ruscheinski J, Wang PL, Ideker T. 2011. Cytoscape 2.8: New features for data integration and network visualization. Bioinformatics 27: 431-432.

Tran EJ, Zhou Y, Corbett AH, Wente SR. 2007. The DEAD-box protein Dbp5 controls mRNA export by triggering specific RNA:protein remodeling events. Mol Cell 28: 850-859.

Trinkle-Mulcahy L, Boulon S, Lam YW, Urcia R, Boisvert FM, Vandermoere F, Morrice NA, Swift S, Rothbauer U, Leonhardt H, et al. 2008. Identifying specific protein interaction partners using quantitative mass spectrometry and bead proteomes. J Cell Biol 183: 223-239.

Tsvetanova NG, Klass DM, Salzman J, Brown PO. 2010. Proteome-wide search reveals unexpected RNA-binding proteins in Saccharomyces cerevisiae. PLOS ONE 5: e12671.

Tutucci E, Stutz F. 2011. Keeping mRNPs in check during assembly and nuclear export. Nat Rev Mol Cell Biol 12: 377-384.

Ule J, Jensen K, Mele A, Darnell RB. 2005. CLIP: A method for identifying protein-RNA interaction sites in living cells. Methods 37: 376-386.

Wang Y, Liu CL, Storey JD, Tibshirani RJ, Herschlag D, Brown PO. 2002. Precision and functional specificity in mRNA decay. Proc Natl Acad Sci 99: 5860-5865.

Wolf JJ, Dowell RD, Mahony S, Rabani M, Gifford DK, Fink GR. 2010. Feedforward regulation of a cell fate determinant by an RNA-binding protein generates asymmetry in yeast. Genetics 185: 513-522.

Received December 23, 2012; accepted in revised form March 7, 2013.

\section{Genome Research}

www.genome.org 


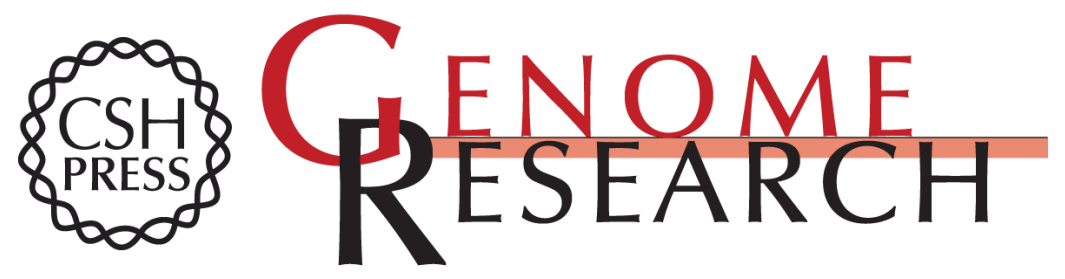

\section{Quantitative proteomic analysis reveals concurrent RNA-protein interactions and identifies new RNA-binding proteins in Saccharomyces cerevisiae}

Daniel M. Klass, Marion Scheibe, Falk Butter, et al.

Genome Res. 2013 23: 1028-1038 originally published online May 1, 2013

Access the most recent version at doi:10.1101/gr.153031.112

\section{Supplemental http://genome.cshlp.org/content/suppl/2013/04/29/gr.153031.112.DC1 \\ Material}

References This article cites 68 articles, 28 of which can be accessed free at:

http://genome.cshlp.org/content/23/6/1028.full.html\#ref-list-1

Open Access Freely available online through the Genome Research Open Access option.

Creative This article is distributed exclusively by Cold Spring Harbor Laboratory Press for the Commons

License first six months after the full-issue publication date (see

http://genome.cshlp.org/site/misc/terms.xhtml). After six months, it is available under a Creative Commons License (Attribution-NonCommercial 3.0 Unported License), as described at http://creativecommons.org/licenses/by-nc/3.0/.

Email Alerting Receive free email alerts when new articles cite this article - sign up in the box at the Service top right corner of the article or click here.

\section{Affordable, Accurate Sequencing.}

To subscribe to Genome Research go to:

https://genome.cshlp.org/subscriptions 